\title{
The Galactic rotation curve from red clump stars
}

\author{
Chao Liu ${ }^{1}$, Glenn van de Ven ${ }^{2}$, Min Fang ${ }^{3}$, Yue $\mathrm{Wu}^{1}$, \\ Kenneth Carrell ${ }^{1}$ and Xiangxiang Xue ${ }^{1,2}$
}

${ }^{1}$ Key Laboratory of Optical Astronomy, National Astronomical Observatories, CAS, 20A

Datun Road, Chaoyang District, 100012, Beijing, China

email: liuchao@nao.cas.cn

${ }^{2}$ Max Planck Institute for Astronomy, Königstuhl 17, D-69117 Heidelberg, Germany

${ }^{3}$ Purple Mountain Observatory and Key Laboratory for Radio Astronomy, Chinese Acedamy of Sciences, 2 West Beijing Road, 210008, Nanjing, China

\begin{abstract}
We use line-of-sight velocities of more than 4000 red clump stars observed from $\mathrm{MMT} /$ Hectospec to derive the Galactic rotation curve between 8 and $13 \mathrm{kpc}$ in Galactocentric radius to better than $10 \mathrm{~km} \mathrm{~s}^{-1}$. A three-component (bulge+disk+halo) with an additional massive ring of $1.66 \times 10^{10} \mathrm{M}_{\odot}$ at about $11.6 \mathrm{kpc}$ provides an excellent fit with the observation.
\end{abstract}

Keywords. Galaxy: disk, Galaxy: kinematics and dynamics, Galaxy: structure

\section{Introduction}

The rotation curve is a powerful tool to map the mass distribution of the galactic disk (Sofue \& Robin 2001). In particular, it is broadly used to detect the dark matter halo around a galaxy. In this work, we use red clump stars, one of the standard candles, in the disk to derive the rotation curve in the outer disk.

We use the line-of-sight velocities of about 4000 red clump stars observed from MMT/Hectospec to measure the rotation curve. The stars are located at $l=100^{\circ}$ $180^{\circ}$ and $b=0^{\circ}$. The mean azimuthal velocities, mean radial velocities and their dispersions at each Galactic radius are estimated from line-of-sight velocities spanned in a broad range of Galactic longitude. Subsequently, the rotation curve is directly estimated from Jeans equation.

Fig 1 shows the derived rotation curve (red line) combined with the HI/CO sources (Sofue et al. (2009)) under the assumption that the local circular speed is $220 \mathrm{~km} \mathrm{~s}^{-1}$. A three-component (Hernquist bulge, exponential disk, and NFW halo) model alone cannot describe the rotation curve, but adding a massive ring of $1.66 \times 10^{10} \mathrm{M}_{\odot}$ at around $R=11.6 \mathrm{kpc}$ provides a perfect fit.

\section{References}

Sofue, Y. \& Robin, V. 2001, ARA $\& A, 39,137$

Sofue, Y., Homma, M., \& Pmodaka, T. 2009, PASJ, 61, 227 